\title{
VENINS COAGULANTS ET ANTICOAGULANTS
}

\author{
PAR \\ 13. - A. Houssay et A. Sondi:I.J
}

(Buenos-Aires)

\begin{abstract}
M Arthus, dont on connait les remarquables travaux sur les 1. venins de serpents, vient de publier plusieurs notes sur l'action qu'ils exercent sur la coagulation sanguine in vitro et in vivo (1). Ayant étudié longuement et d'une façon méthodique ces mêmes problèmes $\left({ }^{2}\right)$, nous tenons à discuter quelques-unes de ses conclusions.
\end{abstract}

ARTHus divise les venins coagulants en deux groupes principaux : les venins à type thrombine (Lachesis lanceolatus, Crotalus terrificus) et les venins à type extrait d'organes (Vipera Russelli). Dans nos expériences nous avons observé que tous ces venins coagulants ont des propriétés de thrombine.

L'action type thrombine signalée par LAMB $\left(^{3}\right)$ et interprétée très correctement par MARTIN (4), a été confirmée par ARTHUS et bien d'autres auteurs, et minutieusement étudiée par nous. Donc il n'y a pas de désaccord sur ce point.

Quant aı venin de Daboïa, qu'ARTHUs considère comme type extrait d'organe, il a aussi une action de thrombine, plutôt faible il est vrai, si on le compare avec d'autres venins. C'est justement avec ce venin que LAMB découvrit le premier exemple d'action coagulante. Donc le venin de Daboïa $\left(^{5}\right)$ a aussi un pouvoir de thrombine.

Si dans des centaines d'expériences, comme c'est notre cas, on établit le pouvoir thrombosant in vivo des venins et on le compare

(1) ARthus M. C. R. Soc. de Biol., 1919, LXXXII, 1156. - Id., 115. Arch. Int. Physiol., 1919, XV, 203.

(2) HOUSSAY et SORDELLI. Anales Soc. Quimica Argentina, 1917, V, 141. C. R. Soc. Biol., 1918, LX X XI, 12. - Rev. Inst. Bacter. Depart. Nac. Higiene, (Bs. As), 1918, I, 485 (note) : 1918, I, 566 (2e note) ; 1919, II, 151. - Rev. Asoc. Med. Argentina, 1919, X X XI, 70. - C. R. Soc. Biol., 1919, LX X X1I, 1029.

(') Scient. Mem. by off. of the Med. and San. Depart. of Gov. of India, 1903, $n^{\circ} 4$.

(1) Journ. Physiol., 1905, X X X II, 207.

(s) Nous avons étudié deux échantillons : l'un provenait du Bombay Bacleriological Laboratory, l'autre du Zoological Garden d'Alipore (par les soins de Sir L. Rogers). 
au pouvoir coagulant in vitro, on s'aperçoit qu'il y a un étroit parallélisme entre les pouvoirs coagulants in vitro, et in vivo.

On peut ériger en règle que seuls les venins à action type thrombine sont capables de produire des coagulations intravasculaires in vivo. Ceci fut démontré être le cas pour 14 des venins que nous étudiâmes.

La seule exception fut celle du venin de Crotalus adamanteus, et encore il est probable qu'elle n'est qu'apparente. Ce venin produit des thromboses quand on l'injecte à de fortes doses, ce qui nous fait supposer qu'il a un pouvoir thrombinique très faible. Si l'on tient compte que c'est l'un des venins qui, mélangés au fibrinogène, l'altérent en très peu de temps, il est très probable que l'altération précoce du fibrinogène devance le temps nécessaire pour que la faible quantité de la substance thrombinique du venin puisse faire coaguler le sang in vitro.

Hors ce cas, nous tenons à le répéter, tous les venins coagulants in vivo le sont aussi in vitro. C'est une affirmation catégorique que nous faisons, car elle repose sur des faits vérifiés maintes fois et dont toutes les preuves ont été publiées.

Quant a ce qui a trait à l'incoagulabilité du sang produite par les venins quand on les injecte chez le chien, nous avons démontré qu'elle obéit à deux mécanismes essentiels :

10 Les venins coagulants à doses convenables produisent peu à peu la précipitation de tout le fibrinogène, de sorte que le sang se défibrine et devient incoagulable.

$2^{\circ}$ Les venins anticoagulants in vitro (et entre eux celui de Crotalus adamanteus) détruisent (lipolyse) le cytozymie et par ce fait empêchent la formation de la thrombine ( $\left.{ }^{1}\right)$. Injectés in vivo ils produisent l'incoagulabilité parce qu'ils détruisent le cytozyme. Il y a une certaine diminution de fibrinogène, généralement très peu marquée.

Il est plutôt rare d'observer l'apparition de l'antithrombine dans les plasmas des animaux envenimés. C'est justement le venin de Crotalus adamanteus qui la fit apparaitre le plus souvent dans nos expériences.

Nous insistons sur ce point, car il contredit l'opinion très en vogue qui attribue à l'antithrombine l'effet anticoagulant des venins. Pour

(1) Ce fait, découvert par Morawitz (1904), confirmé par Mellanby (1909), HiRSCHFELD et KLINGER (1915), fut définitivement démontré par nos expériences (1917). 
démontrer la présence de l'antithrombine, on doit tenir compte de la diminution de fibrinogène dans le sang des animaux injectés avec les venins (Cobra ou autres). Il est surtout nécessaire d'ajouter comparativement de la thrombine déjà préparée aux plasmas oxalatés recueillis avant et après l'injection du venin.

Arthus admet ${ }^{(1)}$ qu'on peut employer à cet effet des venins de Lachesis. Mais nous avons démontré que la thrombine sanguine et les thrombines des venins sont des substances complètement différentes, car l'antithrombine ne neutralise pas les venins coagulants et, par contre, les sérums antivenimeux n'ont pas d'action spéciale sur l'antithrombine sanguine.

La comparaison souvent faite entre la protéotoxie, l'anaphylaxie et l'action des venins repose sur une apparente analogie. Cette notion conduit forcément à des généralisations. Nous démontrerons dans un prochain travail que les effets hypotensifs marqués des venins sont toujours d'accord avec leurs effets coagulants in vivo.

Avant d'aborder les actions des venins in vivo nous avons étudié leurs actions in vitro sur le sang et les plasmas purs ou stables. Ensuite nous avons étudié séparément l'action de chaque venin sur chacun des agents connus qui interviennent dans la coagulation, en les laissant en contact un certain temps ; puis en neutralisant le venin par le sérum antivenimeux approprié, on pouvait rechercher l'état de la substance ainsi traitée.

Cette méthode longue et délicate, mais sûre, nous a permis de trouver des faits d'importance pour interpréter l'action des venins sur la coagulation.

Nous ne nous dissimulons pas que les phénomènes de la coagulation sont évidement très complexes, mais pour le moment l'action des venins qu'Arthus a étudiés peut être comprise de la façon suivante :

Venin de Daboïa. (Vipera Russelli). Ce venin coagule les plasmas citratés ou oxalatés, comme le démontra LamB et comme nous l'avons toujours confirmé. Son action thrombinique est bien plus faible que celle de la plupart des venins coagulants.

Ce venin in vitro altère peu le cytozyme et n'altère ni le sérozyme, ni la thrombine, ni le fibrinogène. Il a la remarquable propriété de

(1) Arch. Intern. de Physiol., 1919, 218. 
produire instantanément la formation de thrombine quand on l'ajoute au mélange sérozyme-cytozyme-calcium, n'ayant d'ailleurs lui-même aucune action de sérozyme ou de cytozyme. Certains de ces faits démontrés par nous ont été confirmés par ARTHus.

Injecté dans les veines, ce venin produit une phase positive intense, qui n'est pas d'accord avec son pouvoir thrombinique (comme c'est aussi le cas pour les venins coagulants, sauf celui de Bungarus fasciatus, qui se comporte comme $V$. Russelli).

Injecté aux animaux à doses convenables, ce venin exerce son action thrombinique, car le sang coagule si on le recueille sur $1 / 10$ vol. de solution d'oxalate sodique à $1 \%$. Il coagule aussi, si on ajoute du sérum normal ou antivenimeux non spécifique, mais ne coagule pas si on ajoute du sérum antidaboïa.

Il faut une dose relativement forte de ce venin (comparativement avec ceux de Lachesis, Crotalus, Notechis, Pseudechis, Echis, etc.) pour produire la coagulation intravasculaire. Ceci est d'accord avec le pouvoir coagulant in vitro comparé du venin de Daboïa et de ces autres venins. (1).

Si on extrait du sang d'un chien et qu'on note le temps qu'il met à coaguler avant et après l'injection intraveineuse de venins coagulants, on observe une accélération passagère (phase positive), suivie d'un retard progressif (phase négative). Nous avons démontré que la phase négative est due à la défibrination progressive du sang ( ${ }^{2}$ ). Quant à la phase positive, elle est due, pour presque tous les venins coagulants, à ce que leur action coagulante s'ajoute au processus normal de coagulation. Mais justement le venin de Daboïa a une faible action coagulante in vitro et in vivo et cependant il produit à petite dose une phase positive très intense et très prolongée. Cette phase positive s'explique comme nous l'avons démontré et ARTHus vient de le confirmer, parce que le venin accélère extraordinairement la formation de la thrombine.

Nous avons fait observer avant ARTHUs que ces effets sont comparables à ceux des extraits d'organes, mais en faisant remarquer que le venin est actif à des doses infimes. Les extraits d'organes ne produisent pas la formation si brusque de la thrombine.

(1) Comparer : Reo. Inst. Depart. Nac. Higiene (Bs. As), 1918, no 5, 570 (action in vilro) et page 17 du volume $11, n^{\circ} 2$ de la même revue (action in vivo).

(2) La précipitation du fibrinogène fut signalée par MELLANBY dans le cas de Echis carinatus. Journ. Physiol., 1909. 
Venin de Cobra (Naja tripudians) Le venin de Cobra est anticoagulant in vitro et in vivo, parce qu'il détruit le cytozyme. Ceci fut démontré par Morawitz (1904), Mellanby (1909) ( ${ }^{1}$ ), HirschFELD et KLINGER (1915) $\left({ }^{2}\right)$ et définitivement par nous (1917-1918).

Nous avons démontré que ce venin détruit notablement le cytozyme, très faiblement le sérozyme, n'altère pas le fibrinogène, empêche la disparition de la thrombine et accélère la coagulation du mélange thrombine-fibrinogène.

In vivo il y a destruction de cytozyme, ce qui fait que le sang extrait des vaisseaux ne forme pas de thrombine. Le fibrinogène diminue, mais il est rare de voir apparaitre de l'antithrombine.

Antagonisme Dabola-Cobra. Il n'y a pas d'antagonisme véritable entre ces deux venins. L'antagonisme apparent que l'on peut observer quand on les ajoute à un plasma qu'ensuite on recalcifie, s'explique facilement, car si le venin de Cobra est ajouté en quantité telle qu'il n'a pas encore détruit tout le cytozyme, celui-ci, en présence du venin de Daboïa, produit hrusquement de la thrombine.

Crotalus Adamanteus. Détruit le cytozyme, attaque faiblement le sérozyme et altère rapidement le fibrinogène.

In vivo il empêche la coagulation, surtout par son pouvoir anticytozymique. Il produit ou non, selon les cas, l'apparition d'antithrombine.

\section{CONCLUSIONS}

1. Les venins de serpents produisent l'incoagulabilité in vivo par delix mécanismes :

a) Les venins coagulants in vitro produisent in vivo la précipitation progressive du fibrinogène et défibrinent le sang.

b) Les venins anticoagulants in vitro détruisent le cytozyme et par cela même empêchent la formation de la thrombine sanguine.

2. Les venins coagulants produisent l'accélération de la coagulation (phase positive) sitôt après leur injection veineuse. Cette accélération est due :

a) Dans presque tous les cas à l'action coagulante du venin qui s'ajoute à la coagulation normale.

b) Pour quelques venins (type Daboïa) il s'ajoute encore la propriété d'accélérer fortement la production de la thrombine sanguine.

(1) Deuls. Arch. Klin. Med., 1904, LXXX, 340.

(2) Journ. of Physiol., 1909, XXXVIII, 441.

(2) Biochem. Zeilsch., 1915, LXX, 399. 